\title{
Correction to: Towards the exact simulation using hyperbolic Brownian motion
}

\author{
Yuuki Ida ${ }^{1}$ (D) Yuri Imamura ${ }^{2}$
}

Published online: 19 July 2018

○ The JJIAM Publishing Committee and Springer Japan KK, part of Springer Nature 2018

\section{Correction to: Japan J. Indust. Appl. Math. (2017) 34:833-843 https://doi.org/10.1007/s13160-017-0265-9}

The authors would like to correct the errors in the publication of the original article. The corrected details are given below for your reading.

Theorem 2 (i) claims that the derivative with respect to $x$ of logarithm of the heat kernel of $\mathbb{H}^{2}$ is bounded, but its proof presented in the paper has the following gap. In Line- 7 of page 837 , it is claimed that,

$$
e^{t}(2 \pi) p_{4}(t, r) \leq \frac{3}{2} \frac{1}{1+\cosh (r)} p_{2}(t, r)
$$

which is implied by Gruet's formula:

$$
p_{n}(t, r)=\frac{e^{-(n-1)^{2} t / 8}}{\pi(2 \pi)^{n / 2} t^{1 / 2}} \Gamma\left(\frac{n+1}{2}\right) \int_{0}^{\infty} \frac{e^{\left(\pi^{2}-b^{2}\right) / 2 t} \sinh (b) \sin (\pi b / t)}{(\cosh (b)+\cosh (r))^{(n+1) / 2}} d b,
$$

where $p_{n}\left(t, r\left(z, z^{\prime}\right)\right)$ is the heat kernel of $\mathbb{H}^{n}, r\left(z, z^{\prime}\right)$ being the hyperbolic distance between $z$ and $z^{\prime}$. However, since the integrand in the left-hand-side of (1) is not always positive, the inequality is not guaranteed.

Actually the claimed inequality is too sharp to prove, we should at this stage admit that Theorem 2 is an error. Instead here we present a corrected version.

The original article can be found online at https://doi.org/10.1007/s13160-017-0265-9.

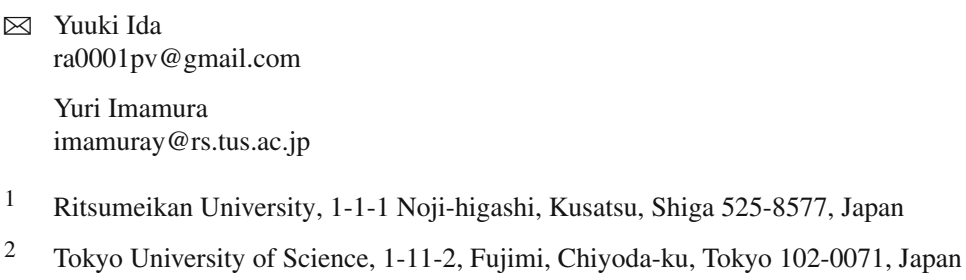


Let

$$
\theta\left(t,(x, y),\left(x^{\prime}, y^{\prime}\right)\right):=\mu(x, y) \frac{\frac{\partial q_{2}}{\partial x}\left(t,(x, y),\left(x^{\prime}, y^{\prime}\right)\right)}{q_{2}\left(t,(x, y),\left(x^{\prime}, y^{\prime}\right)\right)}
$$

Theorem 2 (i) For $z=(x, y), z^{\prime}=\left(x^{\prime}, y^{\prime}\right) \in \mathbb{H}^{2}$, we have that

$$
\left|\partial_{x} q_{2}\left(t, z, z^{\prime}\right)\right| \leq C^{\prime}\left(y y^{\prime}\right)^{-\frac{1}{2}} t^{-\frac{1}{2}} q_{2}\left(2 t, z, z^{\prime}\right)
$$

for some constant $C^{\prime}>0$, and for each $n \geq 2, t>0$ and $\left(s_{1}, \ldots, s_{n-1}\right) \in \Delta_{n-1}(t)$

$$
\mathbb{E}\left[\prod_{i=1}^{n} \theta\left(s_{i}-s_{i-1}, Z_{s_{i-1}}^{0}, Z_{s_{i}}^{0}\right) \mid Z_{0}^{0}=z, Z_{t}^{0}=z^{\prime}\right] q\left(t, z, z^{\prime}\right)\left(y^{\prime}\right)^{-2} \in L^{1}\left(\Delta_{n-1}(t)\right)
$$

where $s_{0}=0, s_{n}=t$.

(ii) Set

$$
h_{1}\left(t, z, z^{\prime}\right)=\mu(x, y) \frac{\partial}{\partial x} q_{2}\left(t, z, z^{\prime}\right)\left(y^{\prime}\right)^{-2},
$$

and

$$
\begin{aligned}
h_{n}\left(t, z, z^{\prime}\right):= & \int_{\Delta_{n-1}(t)} \mathbb{E}\left[\prod_{i=1}^{n} \theta\left(s_{i}-s_{i-1}, Z_{s_{i-1}}^{0}, Z_{s_{i}}^{0}\right) \mid Z_{0}^{0}=z, Z_{t}^{0}=z^{\prime}\right] \\
& \times q_{2}\left(t, z, z^{\prime}\right) /\left(y^{\prime}\right)^{2} d s_{1} \ldots d s_{n-1}
\end{aligned}
$$

for $n \geq 2$. Then, the series $\sum_{n=1}^{N} h_{n}\left(t, z, z^{\prime}\right)$ is absolutely convergent as $N \rightarrow \infty$ uniformly in $\left(t, z, z^{\prime}\right)$ on every compact set.

(iii) The transition density of $Z^{\mu}$ is given by

$$
s\left(t, z, z^{\prime}\right):=\frac{q_{2}\left(t, z, z^{\prime}\right)}{\left(y^{\prime}\right)^{2}}+\int_{\mathbb{H}^{2}} \int_{0}^{t} \frac{q_{2}\left(t-s, z, z^{\prime \prime}\right)}{\left(y^{\prime \prime}\right)^{2}} \Phi\left(s, z^{\prime \prime}, z^{\prime}\right) d s d z^{\prime \prime},
$$

where $\Phi\left(t, z, z^{\prime}\right)=\sum_{n=1}^{\infty} h_{n}\left(t, z, z^{\prime}\right)$.

To prove Theorem 2, we use the following

Theorem [1]. Let $p_{n}$ be the heat kernel of the hyperbolic space $\mathbb{H}^{n}$, and

$$
k_{n+1}(t, r):=(2 \pi t)^{-\frac{n+1}{2}} e^{-\frac{n^{2}}{8} t-\frac{r^{2}}{2 t}-\frac{n r}{2}}(1+r+t)^{\frac{n}{2}-1}(1+r),
$$

for $n \geq 1, t>0$, and $r>0$. For any integer $n>1$, then

$$
p_{n}(t, r) \sim k_{n}(t, r)
$$

uniformly in $r \geq 0$ and $t>0$. 
Proof of Theorem 2 By (4) and (5), we have in particular,

$$
p_{2}(t, r) \sim \frac{e^{-\frac{t}{8}-\frac{r^{2}}{2 t}-\frac{r}{2}}}{2 \pi t}(1+r+t)^{-\frac{1}{2}}(1+r),
$$

and

$$
p_{4}(t, r) \sim \frac{e^{-\frac{9 t}{8}-\frac{r^{2}}{2 t}-\frac{3 r}{2}}}{(2 \pi t)^{2}}(1+r+t)^{\frac{1}{2}}(1+r) .
$$

To start with, we calculate the derivative with respect to $x$ of $q_{2}$. Using Milson's formula, we have

$$
\left|\frac{\partial q_{2}}{\partial x}\left(t,(x, y),\left(x^{\prime}, y^{\prime}\right)\right)\right|=\frac{\left|x-x^{\prime}\right|}{y y^{\prime}} 2 \pi e^{t} p_{4}(t, r) .
$$

Then,

$$
\begin{aligned}
\frac{\left|x-x^{\prime}\right|}{y y^{\prime}} 2 \pi e^{t} p_{4}(t, r) & \leq K \frac{\left|x-x^{\prime}\right|}{y y^{\prime}} 2 \pi e^{t} \frac{e^{-\frac{9 t}{8}-\frac{r^{2}}{2 t}-\frac{3 r}{2}}}{(2 \pi t)^{2}}(1+r+t)^{\frac{1}{2}}(1+r) \\
& =K \frac{\left|x-x^{\prime}\right|}{y y^{\prime}} \frac{e^{-\frac{t}{4}-\frac{r^{2}}{4 t}-\frac{r}{2}-\frac{r^{2}}{4 t}+\frac{t}{8}-r}}{2 \pi t^{2}}(1+r+t)^{\frac{1}{2}}(1+r) \\
& \leq K^{\prime} \frac{\left|x-x^{\prime}\right|}{y y^{\prime}}(1+r+t)^{\frac{1}{2}}(1+r+2 t)^{\frac{1}{2}} \frac{e^{-\frac{r^{2}}{4 t}+\frac{t}{8}-r}}{t} p_{2}(2 t, r) \\
& \leq K^{\prime} \frac{\left|x-x^{\prime}\right|}{y y^{\prime}}(1+r+2 t) \frac{e^{-\frac{r^{2}}{4 t}+\frac{t}{8}-r}}{t} p_{2}(2 t, r) \\
& =\frac{K^{\prime}\left|x-x^{\prime}\right|}{y y^{\prime}} \frac{e^{-\frac{r^{2}}{8 t}}}{r}(1+r+2 t) \frac{r e^{-\frac{r^{2}}{8 t}+\frac{t}{8}-r}}{\sqrt{t}} \frac{p_{2}(2 t, r)}{\sqrt{t}},
\end{aligned}
$$

where we have used (5) twice. We shall estimate the last term of (6). First, we observe that

$$
\begin{aligned}
\frac{\left|x-x^{\prime}\right|}{y y^{\prime}} & =\sqrt{\frac{2}{y y^{\prime}}} \sqrt{\frac{\left|x-x^{\prime}\right|^{2}}{2 y y^{\prime}}} \\
& \leq \sqrt{\frac{2}{y y^{\prime}}} \sqrt{\frac{\left|x-x^{\prime}\right|^{2}+\left(y-y^{\prime}\right)^{2}}{2 y y^{\prime}}} \\
& =\sqrt{\frac{2}{y y^{\prime}}} \sqrt{\cosh (r)-1} \\
& \leq \sqrt{\frac{2}{y y^{\prime}}} \sqrt{\cosh ^{2}(r)-1}=\sqrt{\frac{2}{y y^{\prime}}} \sinh (r) .
\end{aligned}
$$


Then we see that

$$
\frac{\left|x-x^{\prime}\right|}{r \sqrt{y y^{\prime}}} e^{-\frac{r^{2}}{8 t}}
$$

is bounded since $\lim _{r \rightarrow 0} \sinh (r) / r=1$ and $\lim _{r \rightarrow \infty} \sinh (r) e^{-\frac{r^{2}}{8 t}} / r=0$. Noting that $(1+r+2 t) e^{\frac{t}{8}-r}$, and $\frac{r}{\sqrt{t}} e^{-\frac{r^{2}}{8 t}}$ with $(t, r) \in[0, T] \times[0, \infty)$, are bounded, we obtain (2).

Subsequently, we see that $h_{1}$ is bounded by $C q_{2} / \sqrt{y y^{\prime} t}$ with some constant $C$. Therefore,

$$
\begin{aligned}
\mid h_{n} & \left(t, z, z^{\prime}\right) \mid \\
\leq & \int_{\Delta_{n-1}(t)} \mathbb{E}\left[\prod_{i=1}^{n}\left|\theta\left(s_{i}-s_{i-1}, Z_{s_{i-1}}^{0}, Z_{s_{i}}^{0}\right)\right| \mid Z_{0}^{0}=z, Z_{t}^{0}=z^{\prime}\right] \\
& \times q_{2}\left(t, z, z^{\prime}\right) /\left(y^{\prime}\right)^{2} d s_{1} \ldots d s_{n-1} \\
\leq & \int_{\Delta_{n-1}(t)} \int_{\mathbb{H}} \ldots \int_{\mathbb{H}}|\mu(z)|\left|\frac{\partial_{x} q_{2}\left(s_{1}, z, z_{1}\right)}{\left(y_{1}\right)^{2}}\right| \times \cdots \times\left|\mu\left(z_{n-1}\right)\right|\left|\frac{\partial_{x} q_{2}\left(t-s_{n-1}, z_{n-1}, z^{\prime}\right)}{\left(y^{\prime}\right)^{2}}\right| \\
& \times d z_{1} \ldots d z_{n-1} d s_{1} \ldots d s_{n-1} \\
\leq & \int_{\Delta_{n-1}(t)} \int_{\mathbb{H}} \ldots \int_{\mathbb{H}}\left|\mu(z) \times \cdots \times \mu\left(z_{n-1}\right)\right| \\
& \times \frac{q_{2}\left(2 s_{1}, z, z_{1}\right) /\left(y_{1}\right)^{2} \times \cdots \times q_{2}\left(2\left(t-s_{n-1}\right), z_{n-1}, z^{\prime}\right) /\left(y^{\prime}\right)^{2}}{\sqrt{y} y_{1} \ldots y_{n} \sqrt{y^{\prime}} \sqrt{s_{1} \times \cdots \times\left(t-s_{n-1}\right)}} \\
& \times d z_{1} \ldots d z_{n-1} d s_{1} \ldots d s_{n-1} \\
\leq & \int_{\Delta_{n-1}(t)} \int_{\mathbb{H}} \ldots \int_{\mathbb{H}} K_{0}^{n} C^{n} \sqrt{\frac{y}{y^{\prime}}} \frac{q_{2}\left(2 s_{1}, z, z_{1}\right) /\left(y_{1}\right)^{2} \times \cdots \times q_{2}\left(2\left(t-s_{n-1}\right), z_{n-1}, z^{\prime}\right) /\left(y^{\prime}\right)^{2}}{\sqrt{s_{1} \times \cdots \times\left(t-s_{n-1}\right)}} \\
& \times d z_{1} \ldots d z_{n-1} d s_{1} \ldots d s_{n-1} .
\end{aligned}
$$

Using the Chapman-Kolmogorov equation, we then obtain that

$$
\begin{aligned}
\left|h_{n}\left(t, z, z^{\prime}\right)\right| & \leq K_{0}^{n} C^{n} \sqrt{\frac{y}{y^{\prime}}} \frac{q_{2}\left(2 t, z, z^{\prime}\right)}{\left(y^{\prime}\right)^{2}} \int_{\Delta_{n-1}(t)} \frac{1}{\sqrt{s_{1} \times \cdots \times\left(t-s_{n-1}\right)}} d s_{1} \ldots d s_{n-1} \\
& =K_{0}^{n} C^{n} \sqrt{\frac{y}{y^{\prime}}} \frac{q_{2}\left(2 t, z, z^{\prime}\right)}{\left(y^{\prime}\right)^{2}} t^{\frac{n-1}{2}} \prod_{i=1}^{n} \beta\left(\frac{i}{2}, \frac{1}{2}\right) .
\end{aligned}
$$

Here we have in particular shown (3).

If $n=2 m$ with some natural number $m$, we have

$$
\begin{aligned}
\prod_{i=1}^{2 m} \beta\left(\frac{i}{2}, \frac{1}{2}\right) & =\prod_{i=1}^{2 m} \frac{\Gamma\left(\frac{i}{2}\right) \Gamma\left(\frac{1}{2}\right)}{\Gamma\left(\frac{i+1}{2}\right)} \\
& =\prod_{i=2 k, k \in\{1 \cdots m\}} \frac{\Gamma\left(\frac{i}{2}\right) \Gamma\left(\frac{1}{2}\right)}{\Gamma\left(\frac{i+1}{2}\right)} \prod_{i=2 k-1, k \in\{1 \cdots m\}} \frac{\Gamma\left(\frac{i}{2}\right) \Gamma\left(\frac{1}{2}\right)}{\Gamma\left(\frac{i+1}{2}\right)}
\end{aligned}
$$




$$
\begin{aligned}
& =\prod_{k=1}^{m} \frac{\Gamma(k) \Gamma\left(\frac{1}{2}\right)}{\Gamma\left(\frac{2 k+1}{2}\right)} \prod_{k=1}^{m} \frac{\Gamma\left(\frac{2 k-1}{2}\right) \Gamma\left(\frac{1}{2}\right)}{\Gamma(k)} \\
& =\prod_{k=1}^{m}\left(\frac{\left.\Gamma(k) \Gamma\left(\frac{1}{2}\right) \Gamma \frac{\left(\frac{2 k-1}{2}\right) \Gamma\left(\frac{1}{2}\right)}{\Gamma\left(\frac{2 k+1}{2}\right)}\right)}{\Gamma(k)}\right) \\
& =\pi^{m} \prod_{k=1}^{m}\left(\frac{\Gamma\left(\frac{2 k-1}{2}\right)}{\frac{2 k-1}{2} \Gamma\left(\frac{2 k-1}{2}\right)}\right)=\pi^{m} \prod_{k=1}^{m}\left(\frac{1}{\frac{2 k-1}{2}}\right)=\frac{\pi^{m-1 / 2}}{\Gamma\left(\frac{2 m+1}{2}\right)} .
\end{aligned}
$$

On the other hands, when $n=2 m-1$ for any $m \in \mathbb{N}$, we have

$$
\begin{aligned}
\prod_{i=1}^{2 m-1} \beta\left(\frac{i}{2}, \frac{1}{2}\right) & =\prod_{i=1}^{2 m-1} \frac{\Gamma\left(\frac{i}{2}\right) \Gamma\left(\frac{1}{2}\right)}{\Gamma\left(\frac{i+1}{2}\right)} \\
& =\prod_{i=2 k, k \in\{1 \cdots m-1\}} \frac{\Gamma\left(\frac{i}{2}\right) \Gamma\left(\frac{1}{2}\right)}{\Gamma\left(\frac{i+1}{2}\right)} \prod_{i=2 k-1, k \in\{1 \cdots m\}} \frac{\Gamma\left(\frac{i}{2}\right) \Gamma\left(\frac{1}{2}\right)}{\Gamma\left(\frac{i+1}{2}\right)} \\
& =\prod_{k=1}^{m-1} \frac{\Gamma(k) \Gamma\left(\frac{1}{2}\right)}{\Gamma\left(\frac{2 k+1}{2}\right)} \prod_{k=1}^{m} \frac{\Gamma\left(\frac{2 k-1}{2}\right) \Gamma\left(\frac{1}{2}\right)}{\Gamma(k)} \\
& \left.=\frac{\Gamma\left(\frac{2 m-1}{2}\right)}{\Gamma(m)} \prod_{k=1}^{m-1}\left(\frac{\Gamma(k) \Gamma\left(\frac{1}{2}\right)}{\Gamma\left(\frac{2 k-1}{2}\right) \Gamma\left(\frac{1}{2}\right)}\right)^{\Gamma(k)}\right) \\
& =\frac{\pi^{m-1} \Gamma\left(\frac{2 m-1}{2}\right)}{\Gamma(m)} \prod_{k=1}^{m-1} \frac{\Gamma\left(\frac{2 k-1}{2}\right)}{\frac{2 k-1}{2} \Gamma\left(\frac{2 k-1}{2}\right)} \\
& =\frac{\pi^{m-1} \Gamma\left(\frac{2 m-1}{2}\right)}{\Gamma(m)} \prod_{k=1}^{m-1} \frac{1}{\frac{2 k-1}{2}}=\frac{\pi^{m-3 / 2} \Gamma\left(\frac{2 m-1}{2}\right)}{\Gamma(m) \Gamma\left(\frac{2 m-1}{2}\right)}=\frac{\pi^{m-3 / 2}}{(m-1) !}
\end{aligned}
$$

Then,

$$
\begin{aligned}
\sum_{n=1}^{\infty}\left|h_{n}\left(t, z, z^{\prime}\right)\right| \leq & \sum_{n=1}^{\infty} K_{0}^{n} C^{n} \sqrt{\frac{y}{y^{\prime}}} \frac{q_{2}\left(2 t, z, z^{\prime}\right)}{\left(y^{\prime}\right)^{2}} t^{\frac{n-1}{2}} \prod_{i=1}^{n} \beta\left(\frac{i}{2}, \frac{1}{2}\right) \\
= & \sqrt{\frac{y}{y^{\prime}}} \frac{q_{2}\left(2 t, z, z^{\prime}\right)}{\left(y^{\prime}\right)^{2}} \sum_{n=1}^{\infty}\left(K_{0}^{2 n} C^{2 n} t^{\frac{2 n-1}{2}} \prod_{i=1}^{2 n} \beta\left(\frac{i}{2}, \frac{1}{2}\right)\right. \\
& \left.+K_{0}^{2 n-1} C^{2 n-1} t^{n-1} \prod_{i=1}^{2 n-1} \beta\left(\frac{i}{2}, \frac{1}{2}\right)\right) \\
= & \sqrt{\frac{y}{y^{\prime}}} \frac{q_{2}\left(2 t, z, z^{\prime}\right)}{\left(y^{\prime}\right)^{2}} \sum_{n=1}^{\infty}\left(K_{0}^{2 n} C^{2 n} t^{\frac{2 n-1}{2}} \frac{\pi^{n-1 / 2}}{\Gamma\left(\frac{2 n+1}{2}\right)}\right. \\
& \left.+K_{0}^{2 n-1} C^{2 n-1} t^{n-1} \frac{\pi^{n-3 / 2}}{(n-1) !}\right)
\end{aligned}
$$


which shows that the series is convergent.

The proof of (iii) in the paper need not be corrected.

\section{Reference}

1. Davies, E.B., Mandouvalos, N.: Heat Kernel bounds on hyperbolic space and Kleinian groups. Proc. Lond. Math. Soc. (3) 52(1), 182-208 (1988) 